\title{
Territorio, nación y lenguas vernáculas
}

La huella aragonesista del gobierno autonómico bipartito PSOE-CHA (2015-2018)

\section{Michel Martínez Pérez}

\section{Q OpenEdition \\ 1 Journals}

\section{Edición electrónica}

URL: http://journals.openedition.org/agedor/4972

DOI: $10.4000 /$ agedor.4972

ISSN: 2104-3353

\section{Editor}

Laboratoire LISAA

Referencia electrónica

Michel Martínez Pérez, "Territorio, nación y lenguas vernáculas », L'Âge d'or [En línea], 12 | 2019,

Publicado el 01 octubre 2020, consultado el 15 octubre 2020. URL : http://journals.openedition.org/ agedor/4972 ; DOI : https://doi.org/10.4000/agedor.4972

Este documento fue generado automáticamente el 15 octubre 2020.

L'Âge d'or. Images dans le monde ibérique et ibéricoaméricain 


\title{
Territorio, nación y lenguas vernáculas
}

\author{
La huella aragonesista del gobierno autonómico bipartito PSOE-CHA \\ (2015-2018)
}

\section{Michel Martínez Pérez}

1 Las elecciones locales que se celebraron en España en mayo de 2015 confirmaron que el bipartidismo Partido Popular-Partido Socialista Obrero Español, imperante en los últimos 35 años de la democracia española, estaba en crisis, tocado. Tocado, pero no hundido como lo reflejarían las siguientes elecciones generales de 2015 (20-D) que, por primera vez en la historia, se tuvieron que repetir unos meses después, ya en 2016 (26J). En dichos comicios, el PP y el PSOE se mantuvieron como primera y segunda fuerza política, aunque asistieron, impotentes, a la irrupción con fuerza de nuevas formaciones (Podemos y Ciudadanos) que quedaron a poca distancia ${ }^{1}$. A nivel municipal, en mayo de 2015, especialmente en las grandes urbes, Podemos y sus socios locales (las llamadas confluencias), lograron el sorpasso al PSOE, pudiendo constituir “ consistorios del cambio" de diversa índole en Madrid, Barcelona, Valencia, Zaragoza, Cádiz, Santiago, Coruña... Dicho sorpasso, Podemos anhelaba reeditarlo el 20-D, también el 26-J. Para ello, formaron con la Izquierda Unida (IU) de Alberto Garzón, en la primera repetición de unas elecciones generales desde la restauración democrática, la coalición Unidos Podemos, sin que ésta consiguiera su propósito.

2 En Aragón, como en otras 12 comunidades autónomas (CC.AA.) del Estado, las antiguamente llamadas de "régimen común", las elecciones municipales coinciden con las autonómicas. Con los resultados de estos últimos se configuran las Cortes de Aragón, que desde 1983, presenta la singularidad de ser una de las cámaras autonómicas más plurales y multipartidistas del conjunto del Estado (entre 4 y 7 formaciones por legislatura), una característica más propia de la España periférica y de las nacionalidades históricas - con partidos territoriales - que de la España interior - con partidos exclusivamente de ámbito estatal. En Aragón, a la representación parlamentaria de los tradicionales partidos de ámbito estatal (PP, PSOE, e Izquierda Unida) se sumó en 2015 la de Podemos y Ciudadanos. En cuanto a los partidos de ámbito 
no estatal, o territoriales, tanto los aragonesistas conservadores del Partido Aragonés (Par) como los progresistas de Chunta Aragonesista (CHA) mantuvieron su representación parlamentaria en el Palacio de la Aljafería. El eje ideológico izquierda/ derecha cuenta, por tanto en Aragón, con dos partidos territoriales que pueden adscribirse a cada uno de los ejes: el Par (derecha) y CHA (izquierda) ${ }^{2}$.

En 2015, el eje del centro-izquierda salió reforzado con un triunfo electoral contundente. De los 67 diputados con que cuentan las Cortes de Aragón, PSOE, Podemos, CHA e IU sumaron 35, uno más, por tanto, que la mayoría absoluta, frente a los 32 que se anotó el bloque del centro-derecha (PP, Ciudadanos y Par) ${ }^{3}$. Esta mayoría de centro-izquierda en el parlamento fue precisamente la que propició la alternancia política en Aragón después de una legislatura de gobierno PP-PAR (2011-2015). Con el apoyo parlamentario de Podemos e Izquierda Unida (sujeto a los vaivenes de la política municipal y estatal), se fraguó una inédita coalición PSOE-CHA en el ejecutivo aragonés, basada en 50 medidas que acordaron los socialistas y los aragonesistas progresistas de CHA. Para éstos, la entrada en el gobierno aragonés representa un hito que satisface su histórica ambición de gobierno autonómico, aunque con sabor agridulce ya que la formación nacionalista no ha dejado de perder apoyo electoral desde 2003 (pasando de 9 a solo 2 diputados) y se ha visto perjudicada en 2015, en parte por la irrupción de Podemos, hasta verse debilitada como nunca desde 1995, año en que los aragonesistas progresistas consiguieron su primera representación parlamentaria en Aragón.

4 Así pues, ¿qué impronta "aragonesista" está dejando CHA en este gobierno de coalición? ¿No es paradójico que la entrada en el gobierno se produzca en el momento de mayor debilidad del partido desde que entrara en las Cortes en 1995? ¿Puede ser víctima CHA del "abrazo del oso" que ya sufrió después de su experiencia gubernamental en el Ayuntamiento de Zaragoza, entre 2003 y 2007, también con el PSOE? ¿Podrán los conservadores acusar al PSOE de ceder ante presiones nacionalistas? Trataremos de responder a estas y otras preguntas después de hacer un repaso al contexto político aragonés y al aragonesismo político de CHA.

\section{El contexto político aragonés}

5 Desde un prisma geopolítico, Aragón podría observarse como un territorio de transición entre la España periférica, bilingüe, con una implantación importante y presencia parlamentaria de partidos autonomistas y/o nacionalistas (o de ámbito no estatal) y la España interior, monolingüe, con una tendencia al bipartidismo de ámbito estatal. Es cierto que, en Aragón, las lenguas propias (aragonés y catalán) no son oficiales y apenas son habladas por el $5 \%$ de la población, pero son objeto de enseñanza y promoción en las zonas en las que fueron o son predominantes (extremos norte y este del territorio). En cuanto a la conciencia aragonesa (o aragonesista), que, por cierto, no tiene por qué ver con la lengua materna, puede traducirse por la existencia, la implantación territorial y la representación institucional de partidos estrictamente aragoneses (y aragonesistas) ${ }^{4}$. A pesar de no ser hegemónicos y de haber cosechado resultados siempre por detrás de los partidos estatales, estos partidos territoriales han venido cumpliendo desde principios del autogobierno aragonés el papel (imprescindible para la gobernabilidad) de partido bisagra en la mayoría de las instituciones aragonesas (municipios, diputaciones provinciales y ejecutivo autonómico) $)^{5}$. Esta condición de territorio de transición entre periferia e interior 
peninsular ha generado un mapa político propio que respondería también a una conciencia identitaria (aragonesa y/o aragonesista) que llevó a todo el arco parlamentario aragonés, en 2007, a calificar Aragón de "nacionalidad histórica" durante la reforma del Estatuto de autonomía de Aragón. Algunos vieron en aquel acto, una suerte de reparación histórica para con la ciudadanía aragonesa que vio en 1982 cómo, contra todo pronóstico, Aragón se descolgó de las CC.AA. de la vía rápida (artículo 151 de la $\mathrm{CE}$ ) y accedió a la autonomía de régimen común, el de la vía lenta (artículo 143) ${ }^{6}$.

6 Decimos contra pronóstico porque en las primeras elecciones generales después de la muerte de Franco, el 15 de junio de 1977, los electores aragoneses (concretamente los de la circunscripción de Zaragoza) eligieron, entre otros, a dos diputados que representaban siglas de estricto ámbito aragonés: Emilio Gastón por el Partido Socialista de Aragón (PSA, en coalición con el Partido Socialista Popular de Enrique Tierno Galván) e Hipólito Gómez de las Roces por la Candidatura Aragonesa Independiente de Centro (CAIC), embrión del Partido Aragonés Regionalista (PAR). En aquellos comicios históricos, los electores aragoneses se convirtieron en los únicos ciudadanos españoles (tras catalanes y vascos) en llevar a representantes de formaciones de ámbito no estatal al Congreso de los Diputados. Más adelante, el PSA acabaría integrándose en el PSOE (1979-1982) o creando Unió(n)/Chunta Aragonesista (1986) mientras que el PAR alcanzaría la presidencia del gobierno autonómico en 1987, en coalición con Alianza Popular. De hecho, el PAR consiguió mantenerse en el poder autonómico de manera casi ininterrumpida hasta 2015, tanto con el PSOE como con el PP.

7 Desde la restauración de la democracia en España, los partidos aragoneses han sido clave para la gobernabilidad de las instituciones aragonesas en todas las legislaturas ya que los partidos estatales no han obtenido nunca mayorías absolutas en solitario, al revés de lo ocurrido en las CC.AA. bipartidistas del centro peninsular (Castilla-La Mancha, Castilla y León o la Comunidad de Madrid), aunque también de algunas nacionalidades históricas periféricas (Comunidad Valenciana o Andalucía). Decíamos más arriba que los partidos territoriales nunca fueron hegemónicos en Aragón, pero tendríamos que matizar. Es cierto que el PAR o CHA nunca han ganado unas elecciones autonómicas (sí municipales en sus respectivos feudos). Sin embargo, a finales de los años 1980, en un momento de recomposición política de la derecha española, el PAR consiguió ser primera fuerza del bloque conservador en Aragón (por delante de Alianza Popular y del Centro Democrático y Social). Tanto es así que los aragonesistas conservadores (regionalistas confesos, que no nacionalistas en aquel momento) pudieron ostentar la presidencia de la Diputación General de Aragón (DGA) en la segunda y tercera legislatura autonómica (1987-1991 y 1991-1993).

8 La opción conservadora del PAR tuvo, pues, cierto éxito en 1987, un tiempo en el que las fuerzas de la derecha española estaban en recomposición (intento de CDS para paliar el hundimiento de UCD y lenta consolidación de AP, luego PP). El Estado de las autonomías, por su parte, se hallaba en pleno proceso de construcción con evidente entusiasmo autonómico en Aragón (1976-1978). La euforia dejó paso a cierta frustración con la aprobación de un Estatuto de régimen común por la vía lenta (1982) y el jacobinismo asumido del primer gobierno aragonés del PSOE (1983-1987) que sofocó cualquier atisbo aragonesista (republicano-federalista) que pudiera aportar el ex-PSA a sus filas (sector del que provenía, para más inri, el propio presidente Santiago 
Marraco $)^{7}$. El socialismo español de la época, aquella recién estrenada socialdemocracia post-marxista (1979-1982) se reivindicaba federal (y federalista) pero la acción de Alfonso Guerra en una modesta federación como la aragonesa acabó definitivamente con el sueño "a la catalana" de un PSA federado al PSOE. Frente al centralismo de un PSOE aragonés, subordinado a la Ejecutiva federal (Ferraz), el PAR logró presentarse como una fuerza exclusivamente aragonesa, regionalista, aunque claramente conservadora sin que pudiera producirse, por tanto, un trasvase de votos entre el electorado del ex-PSA hacia el PAR, cuyo fundador era el último presidente franquista de la Diputación Provincial de Zaragoza ${ }^{8}$. El regionalismo del PAR no iba más allá de un españolismo localista, un autonomismo bien entendido en el nuevo orden constitucional que jamás cuestionaría el marco estatal ni el régimen monárquico ${ }^{9}$. El PAR pudo criticar con facilidad el centralismo del PSOE en el gobierno autonómico y nacional y captar un voto regionalista (conservador y huérfano de otras propuestas políticas de ámbito estatal) que deseaba, por agravio comparativo, alcanzar la autonomía de las grandes naciones culturales inmediatas, Cataluña y País Vasco, o emular el "foralismo" navarro ${ }^{10}$. Más adelante, cuando el Partido Popular se establece como gran partido del centro derecha, fagocita con facilidad el regionalismo aragonés con el que ha gobernado (1987-1991 y 1991-1993) gracias también a la pujanza de las siglas PP a nivel estatal. Después de una sangría de votos al haber fracasado la pretensión del PAR de representar al PP en Aragón (como Unión del Pueblo Navarro UPN - en la comunidad foral) el PAR inicia un viraje hacia el nacionalismo conservador para tratar de convertirse en el PNV o en el CiU de Aragón ${ }^{11}$. Son tiempos en los que se prevé culminar el desarrollo autonómico del Estado que pasa por reformar el Estatuto de autonomía (1996) para equipararse en competencias (educación, sanidad, medios de comunicación públicos) a las CC.AA. del artículo 151 y en denominación: Aragón se convierte en "nacionalidad" por lo que el PAR deja de ser "regionalista" para asumirse como "nacionalista"12. En el ámbito progresista, así se califica ya el otro partido aragonesista que, desde la izquierda, ha conseguido, en 1995, entrar en las Cortes de Aragón: Chunta Aragonesista (CHA) ${ }^{13}$.

\section{El aragonesismo político de Chunta Aragonesista}

9 Chunta Aragonesista (CHA) nace en 1986 en un momento en el que los electores huérfanos del PSA - que no se reconocieron en el PSOE - y los jóvenes de movimientos antimilitaristas, pacifistas, ecologistas, culturales volcados en la protección de las lenguas minorizadas de Aragón (el aragonés y, en menor, medida el catalán), convergen hacia la creación de un partido político aragonesista, socialista, federalista, nacionalista, ecologista y republicano. A grandes rasgos, podría decirse que los fundadores de CHA han vivido una desilusión autonómica y un profundo desencanto con el PSOE - del que siempre desconfiaron - entre otras cosas por el viraje de la OTAN. Aquel año, parece que la única esperanza sólo puede venir de Europa con el ingreso en la CEE que promete, a medio plazo, eliminar fronteras para los aragoneses en tanto que ciudadanos españoles.

10 Así, en 1986, para los aragonesistas progresistas, el Estatuto de 1982 deja mucho que desear y cunde la sensación de haber perdido el tren de la autonomía real y que Aragón ha sido relegado (injustamente según ellos) a una España de "segunda división". Estos fundadores de CHA piensan que el autogobierno aragonés carece de los márgenes de 
maniobra (competencias) para luchar por unas mejores comunicaciones - viarias y ferroviarias - y contra la despoblación y demás problemas endémicos de Aragón, que siguen dependiendo de la buena voluntad del gobierno central de turno. Según los autonomistas, los diputados de las circunscripciones aragonesas que representan partidos de ámbito estatal, estarían más preocupados por la cohesión de su grupo parlamentario y por intereses partidistas que por los intereses de Aragón que podrían, además, estar contrapuestos a los primeros. Según los aragonesistas, la ley electoral española, la división provincial de las circunscripciones electorales y el reparto de escaños proporcional a la población, favorecen a los territorios más poblados al convertirse en electoralmente estratégicos para los partidos de ámbito estatal por su número de diputados. El peso demográfico, y por tanto político de Aragón en el conjunto de España, es residual al no tener la posibilidad aritmética de inclinar una mayoría alternativa incluso si todos los diputados fueran de un solo partido ${ }^{14}$.

11 Cuando CHA irrumpe en el paisaje político aragonés, obtiene unos primeros resultados muy discretos en los comicios locales de 1987, un año después de su creación ${ }^{15}$. En 1991, consigue representación municipal en varias localidades (sobre todo en el Altoaragón pirenaico y prepirenaico, de nuevo, pero también en Teruel, la primera capital de provincia con representación consistorial de CHA. En 1995, los nacionalistas consiguen entrar en las Cortes de Aragón con dos diputados. En 1999 y 2003, el ascenso de CHA en la representación municipal y autonómica es muy notable ${ }^{16}$. Los gobiernos de José María Aznar (PP) favorecen la movilización de los aragonesistas progresistas en torno a CHA. En 2000, el cantautor José Antonio Labordeta es elegido diputado en el Congreso de los diputados, un hito para la formación nacionalista que suplanta así al Par en la representación del aragonesismo político en Madrid ${ }^{17}$. En 2003, CHA logra también el sorpasso aragonesista al Par en las Cortes de Aragón (9 diputados frente a 8). Después de aquellos comicios, CHA podría haber formado un gobierno de coalición con el PSOE que dirigía entonces el catalanohablante Marcelino Iglesias. Sin embargo, el presidente aragonés más longevo, prefirió mantener una coalición de gobierno centrista con el Par (1999-2011) y no tener que asumir las condiciones de los nacionalistas aragoneses en un contexto de crispación entre el nacionalismo español (desacomplejado con la mayoría absoluta del PP) y los nacionalismos periféricos (especialmente el vasco y en menor medida, en aquel momento, el catalán).

En 2007, CHA no consigue mantener los casi cien mil votos obtenidos en 2003. El batacazo es especialmente llamativo en la capital, Zaragoza, donde CHA ha ostentado el gobierno municipal, en coalición con el PSOE de José Alberto Belloch, y no sobrevive al temido "abrazo del oso", ni al "efecto Zapatero" ${ }^{18}$. Paradójicamente, los resultados en ciudades medias de Aragón son mejores que cuatro años antes, demostrando así que el trabajo de CHA para salir de Zaragoza y dejar de verse como un partido esencialmente urbano y joven, empezaba a dar sus frutos ${ }^{19}$. Sin embargo, los nacionalistas no han conseguido hasta la fecha revertir la sangría de votos de 2007 aunque han podido mantenerse como fuerza parlamentaria y municipal en los principales ayuntamientos aragoneses. Tanto es así que, en 2015, a pesar de la irrupción de Podemos, que mermó aún más la maltrecha salud de los aragonesistas de izquierdas, con sólo dos diputados (los mismos que en 1995), los nacionalistas consiguieron formar un gobierno de coalición con el PSOE. 


\section{Huella aragonesista en el gobierno bipartito PSOE- CHA (2015-2017)}

13 Con los resultados que arrojan los comicios locales de 2015 (autonómicos y municipales en Aragón), los dos diputados de CHA en el Palacio de la Aljafería se vuelven determinantes para orquestar una alternativa de izquierdas (PSOE, Podemos, CHA e IU) tras una legislatura conservadora (PP-Par) que se encargó de aplicar los recortes más acusados de la democracia tras la crisis de 2008. En 2015, para revertir esta situación, y acabar con el "austericidio" post-crisis, los nacionalistas aragoneses, liderados por el presidente de CHA, José Luis Soro, vislumbran la hora de las responsabilidades gubernamentales y pactan con el PSOE su entrada en un ejecutivo de progreso para acabar con los recortes, generar un mejor reparto de las riquezas y un gasto más social. Así es, en todo caso, como siempre se ha querido presentar este partido aragonesista, como un partido de gobierno y de izquierdas. La debilidad parlamentaria de CHA pasa desapercibida gracias al pacto de gobierno forjado con el PSOE, ejecutivo que apoyan Podemos e IU desde las Cortes de Aragón.

14 PSOE y CHA (que ya gobernaron en coalición el Ayuntamiento de Zaragoza entre 2003 y 2007) son partidos que se reclaman de la tradición socialista y que han podido evolucionar hacia posiciones socialdemócratas, también en situaciones de gobierno. Representan, por tanto, dos fuerzas políticas compatibles, aunque se disputan el mismo electorado, el de centro izquierda. En los comicios autonómicos, CHA tiene consolidado desde 1995 un colchón de unos 40000 votos, el de los electores aragonesistas, los que José Antonio Labordeta había identificado como los más "nacionalistas" que siempre serían fieles a las siglas de CHA y que no votarían nunca al PSOE ni a IU, por ser propuestas de ámbito estatal. En 2003, CHA pudo beneficiarse, en cambio, de parte de los votos que iban habitualmente al PSOE o IU. CHA recibió por tanto el "voto prestado" de votantes del PSOE desilusionados, que veían que su partido estaba en reconstrucción, noqueado por la mayoría absoluta del PP. CHA se ganó a pulso los sufragios de los más autonomistas, del sector más a la izquierda, también de los más antitrasvasistas, que habían observado que, tanto PSOE como PP, habían planeado trasvases del Ebro desde su delta hacia tierras más meridionales. También recibieron los votos de electores de IU, partido que no consiguió más de un diputado entre 1999 y 2011, convirtiéndose CHA en el "voto útil" para la izquierda del PSOE. Sin embargo, en 2007, los electores prestados del PSOE (en menor medida de IU) volvieron a sus siglas aprovechando la presidencia de José Luis Rodríguez Zapatero y el buen balance de su primera mitad de legislatura (2004-2006) principalmente en los avances sociales y en la derogación del trasvase del Ebro previsto por el último gobierno de Aznar.

15 Así pues, en 2015, los nacionalistas y los socialistas forjaron un pacto de gobierno en torno a 50 puntos que marcarían la agenda de la legislatura ${ }^{20}$. José Luis Soro entró en el ejecutivo presidido por Javier Lambán como Consejero de Vertebración del Territorio, Movilidad y Vivienda. CHA consiguió, por tanto, una sola consejería, aunque relevante para las infraestructuras y la movilidad, unos ámbitos determinantes para tratar de ganarle el pulso al gran reto de la despoblación ${ }^{21}$. 


\section{Territorio}

16 gobierno autonómico. La cartera de Vertebración, Territorio, Movilidad y Vivienda resultó ser una cartera inédita, relativamente amplia, consecuencia del número reducido de consejeros/as ${ }^{22}$ del nuevo gobierno aragonés. La vertebración del territorio responde a un área que siempre ha estado en la lista de reclamaciones (¿agravios?) de CHA para con los gobiernos autonómicos y estatal. Las reclamaciones de mejoras en las carreteras, de liberación de peajes en la que fue durante años la única vía de alta capacidad en Aragón (AP68), la construcción de nuevos ejes viarios para mejorar comunicaciones estratégicas para la movilidad de ciudadanos y mercancías y también la defensa del ferrocarril para vertebrar el territorio aragonés desde Canfranc hasta Sagunto (Valencia) pasando por las tres capitales de provincia aragonesas, así como ciudades intermedias (Cariñena, Calamocha). La apuesta por un ferrocarril de calidad que pusiera por ejemplo a una hora escasa Teruel de Zaragoza (180 kilómetros) ha sido un objetivo político de los nacionalistas (y de otros partidos) para luchar contra la despoblación, consecuencia del éxodo rural que condujo a la máxima de "Zaragoza contra Aragón" o "Zaragón" como se denomina el exceso de concentración de poderes en la capital aragonesa. Para fijar población fuera de Zaragoza, para que exista la igualdad de oportunidades en todo el territorio aragonés (más grande que Bélgica, pero con apenas 1,3 millones de habitantes), se precisa un buen sistema de comunicación ferroviaria entre los núcleos de población. Sin embargo, la realidad del tren en Aragón está muy lejos de cumplir con este propósito. El ferrocarril no llegó a Teruel hasta el año 1901, ya entrado el siglo XX, más tarde, por tanto, que en las demás capitales de provincia. Aún hoy, Teruel es la única capital de provincia española en no tener conexión directa con Madrid y la vía que atraviesa la provincia por el corredor del Jiloca, es de vía única, sin electrificar, y no puede competir en duración de viaje con el transporte privado ${ }^{23}$. En resumidas cuentas, el tren no es competitivo en Aragón, ni siquiera para las mercancías ${ }^{24}$, y se necesitaría una inversión multimillonaria, tal y como lo lleva recordando la coordinadora "Teruel existe" desde 1999.

Durante esta legislatura, el consejero Soro ha podido mostrar cierto dinamismo en torno a este proyecto de vertebración ferroviaria de Aragón que queda englobado en un proyecto mayor, estratégico para las comunicaciones interterritoriales de España y de la Unión Europea: el corredor Cantábrico-Mediterráneo entre Zaragoza y la Y vasca ${ }^{25}$. Sin embargo, no se ha podido evitar una gran decepción en el gobierno de Aragón, y en la sociedad aragonesa en general, al observar que se había "descolgado" de la propuesta de revisión del Mecanismo Conectar Europa (CEF) de la Comisión Europea, la parte Zaragoza-Sagunto (vía Teruel) de este corredor Cantábrico-Mediterráneo. Aun así, las sociedades aragonesa y valenciana, tanto los gobiernos y toda la clase política como el mundo empresarial y la sociedad civil, están organizándose para ejercer una gran presión social sobre el actual Ministerio de Fomento (que dirige un valenciano, José Luis Ábalos) para que España defienda ante la Comisión Europea la inclusión de este tramo ${ }^{26}$. Los puertos de Valencia y Sagunto, como las autoridades de la Comunidad Valenciana, también han apoyado explícitamente esta infraestructura, calificándola incluso de "irrenunciable", a pesar de la competencia y la pugna con otro corredor, el mediterráneo (Algeciras-Barcelona vía Alicante y Valencia) que defiende un potente lobby catalán ${ }^{27}$. Y es que el corredor Cantábrico-Mediterráneo por Teruel no sólo podrá 
conectar los puertos de Valencia y Sagunto al de Bilbao a través del puerto seco de Zaragoza (conectado a Barcelona y Tarragona) sino que además podría enlazar con otro proyecto estrella de la Consejería dirigida por los nacionalistas: la reapertura del Túnel de Canfranc y la consiguiente salida a Francia por Aragón de viajeros y mercancías. El gobierno aragonés, a través de José Luis Soro, ha apostado claramente por este proyecto de reapertura del Canfranc que permitió durante años el paso por el Pirineo central de mercancías y viajeros (1928-1970). La estación internacional de Canfranc, joya arquitectónica de los años 1920 y abandonada desde principios de los años 1970 (el accidente que cortó el tránsito de trenes tuvo lugar en marzo de 1970) está siendo renovada y es objeto de un gran proyecto turístico que simbolizará un paso más en la permeabilización del Pirineo y de las comunicaciones transfronterizas. De hecho, el proyecto de reapertura del paso por Canfranc ha contado con la ayuda política de la región francesa de Aquitania que ha invertido ya millones de euros en la línea OloronBedous, como paso previo a la reapertura del paso central por Aragón. Parece que todas las administraciones (regionales, nacionales y europeas) apoyan este proyecto de nuevo paso transfronterizo que se justifica con la rápida saturación de los pasos de alta capacidad vasco y catalán por Irun y La Jonquera respectivamente.

Para un dirigente aragonesista como José Luis Soro, volver a conectar Aragón con Europa es, por supuesto, un proyecto de inmenso calado para potenciar la economía aragonesa y un símbolo de gran magnitud para el imaginario colectivo aragonés, además de una lucha histórica del pueblo aragonés que he reivindicado la apertura de este paso sin cejar en su empeño, a pesar del nulo interés del franquismo, primero, y de los gobiernos democráticos luego. Es evidente que, desde el aragonesismo político, hacer territorio es también hacer nación. Así lo ha querido aprovechar el primer consejero nacionalista en un gobierno autonómico.

\section{Nación}

La presencia de un partido nacionalista como CHA en el gobierno de Aragón, se ha podido observar en algunas líneas de actuación de la consejería que ostenta José Luis Soro, sobre todo en lo simbólico. Una de las áreas que quedaron bajo su autoridad fue precisamente la cartera de Turismo, una de las primeras competencias que fueron transferidas a Aragón en el proceso autonómico. Han sido varios los ejemplos de "hacer país" que se han desarrollado desde la dirección general de turismo, un área destinada a promover Aragón fuera de sus fronteras, también fuera de España, y que supone, por tanto, una magnífica herramienta para poner Aragón en el mapa, asentar notoriedad y poder citar Aragón sin tener que usar zonas geográficas como Pirineo central... Una de las actuaciones fue la promoción del tramo aragonés del Camino de Santiago, gran reclamo turístico donde los haya. El Camino aragonés ha querido verse desde la dirección general de Turismo, como una metáfora del abandono de Aragón durante décadas y como una sucesión de oportunidades perdidas. El tramo aragonés, como rama del camino francés, está infra-frecuentado por peregrinos y caminantes sobre todo si se compara con el camino navarro, el más conocido, el más transitado y el mejor acondicionado para recibir a las decenas de miles de visitantes cada año. Acondicionar y dar a conocer el tramo aragonés, se planteó como algo esencial para los responsables nacionalistas que querían poner fin a décadas de desaprovechamiento de Aragón como reclamo turístico a pesar de contar con parte del Camino francés de Santiago. Otro 
asunto, en clave interna, y más simbólico que el primero, ha sido la promoción de Aragón como destino turístico entre los propios aragoneses. De la necesidad de conocer bien el territorio (la nación) para estimarlo, divulgarlo y estar orgulloso de la tierra en la que se vive, la dirección general de Turismo hizo unas cuñas radiofónicas de promoción en las que, al referirse a Aragón, se decía "conoce tu país y cuéntalo" 28 . Además de referirse a Aragón como "país" desde la administración autonómica, es de notar también la voluntad de fomentar el orgullo de país como reclamo turístico entre los propios aragoneses para que se conviertan en embajadores de un territorio de interior, a veces olvidado, injustamente ya que concentra maravillas culturales, paisajísticas y gastronómicas, como el mayor número de "pueblos más bonitos de España"29.

Otro asunto en el que el consejero Soro y sus compañeros de partido han podido desplegar el ideario aragonesista, nacionalista, es en otro ámbito simbólico pero cargado de fuerza política. En esta legislatura, los nacionalistas convencieron a sus socios del PSOE, a otras fuerzas de izquierdas (Podemos e IU), y también a los regionalistas conservadores del Par, para aprobar la Ley de Derechos Históricos ${ }^{30}$. Esta ley, aprobada en julio de 2018, causó gran revuelo mediático, sobre todo en la prensa conservadora (Heraldo de Aragón, ABC, El Mundo...) al pretender, entre otras cosas, obtener del Estado la derogación de los decretos de Nueva Planta (1707 en Aragón). Esta ley, que obtuvo el consenso de todas las fuerzas representadas en las Cortes de Aragón (excepto PP y Ciudadanos), se quiso ver desde los sectores "españolistas" como un " trágala" nacionalista de CHA hacia el PSOE, a pesar del españolismo asumido del Presidente Lambán, sobre todo en el delicado contexto del procés independentista catalán. Según los dirigentes y cargos públicos de $\mathrm{CHA}$, esta ley sólo pretende recordar la personalidad jurídica, foral, propia de Aragón, su pasado como "Estado independiente" durante siete siglos, su papel en la confederación avant la lettre que llevó su nombre ${ }^{31}$. Esta ley, parece obvio que también pretende poner Aragón en el mapa de las nacionalidades históricas del Estado español, en el conjunto de las comunidades forales a las que la Constitución de 1978 trató de dar protección y legitimidad por motivos históricos pero cuya autonomía no creó ex nihilo. Aragón se presenta como "nacionalidad", término constitucional, al que se acuña el adjetivo de "histórica". Se habla en la ley de "sucesivas generaciones de aragoneses y aragonesas que fueron construyendo una nación" . Se podrá criticar una visión un tanto esencialista de la historia de Aragón, pero hay que ubicarla, en mi opinión, en la voluntad de reparar a Aragón por la pérdida del tren de la autonomía rápida en 1980. Una reparación que pretende volver a encauzar Aragón en el seno de las autonomías periféricas, históricas, con perfil identitario fuerte (traducido en voto aragonesista desde 1977), y con perfil plurilingüe, a pesar de la minoría lingüística de catalanohablantes y fabláns de l'aragonés y, sobre todo, a pesar de la ausencia de oficialidad de las lenguas minoritarias y minorizadas en Aragón.

\section{Lenguas vernáculas}

21 Aragón presenta una anomalía al constar de dos lenguas propias (una de ellas exclusivamente aragonesa) que no gozan de carácter oficial, ni siquiera en las comarcas o localidades en las que son predominantes entre los ciudadanos. Oficialmente, por tanto, Aragón es una comunidad monolingüe en la que sólo la lengua oficial del Estado, 
el castellano, es también la lengua oficial de la comunidad. Sin embargo, la autonomía aragonesa, desde el primer Estatuto de 1982, es consciente de que ciudadanos aragoneses practican, además del castellano, unas "modalidades lingüísticas" propias. El largo camino hacia la cooficialidad no ha concluido aún, pero muchas iniciativas se han sucedido en Aragón hasta obtener una suerte de oficialidad oficiosa. La Ley de Lenguas de 2009, pactada ya entre PSOE y CHA, supuso un avance, por ejemplo, en el reconocimiento de la calidad de "lenguas de Aragón" tanto del aragonés como del catalán propio del Aragón oriental, la llamada "Franja". Aquella ley acabó con las perífrasis ("modalidades lingüísticas") y con la incógnita de la identidad de dichas lenguas (dudas filológicas sobre la calidad lingüística del "aragonés” y sobre el estándar propuesto por las asociaciones de hablantes y dudas políticas sobre la catalanidad de la lengua de los aragoneses fronterizos con Cataluña y la provincia de Castellón). En 2013, bajo el gobierno conservador (PP-Par) aquel avance se frenó en seco y las lenguas de Aragón conocieron una involución en su protección y promoción, justificada en los recortes presupuestarios. En cuanto a la identidad, también se modificó al adoptar unas perífrasis polémicas para denominar las lenguas propias: Lengua Aragonesa Propia de las áreas Pirenaica y Prepirenaica y Lengua Aragonesa Propia del Área Oriental ${ }^{32}$. En 2015, con el regreso al poder de los partidos de la Ley de Lenguas de 2009, no se derogó la ley de 2013, recurrida ante el Tribunal Constitucional por 63 diputados del Congreso aquel mismo año, entre los cuales el de CHA, Chesús Yuste ${ }^{33}$. Sin embargo, en 2016, el TC desestimó aquel recurso a pesar de que la Ley de lenguas no nombrara explícitamente a las lenguas propias de Aragón ${ }^{34}$. En otras leyes, en cambio, como la del patrimonio aragonés de 1999, sí se viene afirmando explícitamente que aragonés y catalán son las lenguas propias de la comunidad ${ }^{35}$.

A pesar de estos enredos lingüísticos, donde aparecen dos bloques políticamente opuestos, y donde parece que aceptar la catalanidad lingüística de una parte de Aragón equivaldría casi a una cesión territorial, el gobierno PSOE-CHA tomó la decisión de crear la primera Dirección General de Política Lingüística de Aragón, uno de los puntos clave del pacto de investidura y de gobierno entre ambos socios. Esta primera Dirección de la historia de la Diputación General de Aragón ${ }^{36}$, la asumió el jurista y activista cultural y lingüístico, José Ignacio López Susín. Fue uno de los socios fundadores de CHA en 1986, proveniente de la asociación Rolde de Estudios Aragoneses (REA) y Consello d'a Fabla Aragonesa, dos asociaciones creadas en 1977 y 1976 respectivamente, próximas en aquel momento al PSA de Emilio Gastón y José Antonio Labordeta. Es fácil observar en su trayectoria la lucha de los aragonesistas por el reconocimiento, en primer lugar, de un Aragón autónomo en el que, al principio, se pensaba que las lenguas aragonesa y catalana, propias de Aragón, iban a gozar de un régimen de cooficialidad, al menos en las comarcas y localidades donde se practican de manera predominante. A pesar de que las lenguas propias carezcan a día de hoy de carácter oficial en Aragón ${ }^{37}$, las lenguas se enseñan de manera optativa en los centros de enseñanza con notable éxito y la Ley de Lenguas prevé que la administración local pueda tramitar correspondencias y documentos en la lengua propia si así lo solicitara un ciudadano. En cuanto a la Dirección General de Política Lingüística, partiendo de que las lenguas no son oficiales, sí ha podido trabajar sobre diferentes ejes.

23 En una primera parte de la legislatura, el equipo de la Dirección General de Política Lingüística, trató de revertir la involución en política lingüística que supuso la Ley de 2013 y los recortes del gobierno conservador que llevaron a la suspensión de 
convocatorias de premios literarios en las lenguas propias, la cancelación de visitas de autores a las escuelas con optativas de aragonés o catalán, unos proyectos de acercamiento del público escolar a las lenguas locales, que se iniciaron en 1984 bajo el primer gobierno de la (aún tímida) autonomía aragonesa gracias al empeño de José Ramón Bada, consejero de cultura de aquel primer gobierno autonómico, catalanohablante. También se creó una página web oficial para dar a conocer las diferentes tareas llevadas a cabo por esta Dirección General y para dejar un rastro de las campañas de promoción de las lenguas propias de Aragón (vídeos, cuñas radiofónicas...). A nivel educativo también, se ha hecho una importante labor para regular la certificación (siguiendo el marco europeo común de referencia) de los conocimientos del aragonés, equiparando dicha lengua a las demás estudiadas y certificadas en las Escuelas oficiales de Idiomas de la Comunidad Autónoma. A nivel institucional, ha sido destacada la entrada de Aragón entre las seis comunidades oficialmente bilingües en el marco de cumbres multilaterales, además de Asturias.

\section{Conclusión}

Tal y como se ha tratado de demostrar, el primer ejecutivo de coalición PSOE-CHA está dejando una huella aragonesista nada desdeñable. Si bien, Marcelino Iglesias, presidente socialista de la DGA en coalición con el Par (1999-2011) presentaba un perfil federalista y no dudaba en referirse a Aragón como al "país", Javier Lambán representa un PSOE mucho más jacobino, españolista dirán algunos, y no duda en aprovechar el conflicto catalán para resaltar la españolidad y la lealtad constitucional de Aragón, convertido en el "cordón sanitario" frente al independentismo. Sin embargo, los nacionalistas de CHA, a pesar de contar sólo con dos diputados y un sol consejero (José Luis Soro), han podido arrastrar a su socio de gobierno (incluso a las demás fuerzas de izquierdas y a los aragonesistas conservadores) hasta la aprobación de una ley como la de Derechos históricos, muy ambiciosa en el ámbito del reconocimiento nacional de Aragón en el marco de un Estado, el español, asumido plenamente como plurinacional.

En esta legislatura, como ya se hiciera en 2007 con la reforma del Estatuto de autonomía que consagró a Aragón como "nacionalidad histórica", la coalición progresista y aragonesista (PSOE-CHA) ha avanzado claramente en el reconocimiento de Aragón como una nación ibérica con historia propia, una comunidad foral, un hecho diferencial, en definitiva, basado en la historia y la tradición jurídica. Las lenguas propias, por su parte, otra seña de identidad, no parecen estar en el centro de la construcción nacional de Aragón. A pesar de su protección y promoción por el actual gobierno, siguen careciendo de carácter oficial. Al revés de lo que ocurre en otras naciones culturales del Estado, es evidente que las lenguas propias de Aragón, ni siquiera el aragonés, exclusivo de este territorio, no son el pilar del discurso nacionalista ni de la construcción nacional aragonesa en tanto que "nacionalidad histórica" del Estado español. 


\section{NOTAS}

1. https://resultados.elpais.com/elecciones/generales.html Página consultada el 21 de mayo de 2018.

2. Òscar Barbera Aresté, Àstrid Barrio López y Juan Rodríguez Teruel, "Los partidos de ámbito no estatal en Aragón: el Partido Aragonés y la Chunta Aragonesista”, Papers: Revista de sociología, $\mathrm{n}^{\circ}$ 92, Barcelona, 2009, p. 171-195.

3. https://resultados.elpais.com/elecciones/2015/autonomicas/02/index.html Página consultada el 21 de mayo de 2018.

4. Exceptuando el caso de Canarias, todas las CC.AA. con representación parlamentaria nacionalista son territorios periféricos con lengua propia, elemento que puede presentarse como pilar del hecho diferencial reivindicado por el movimiento particularista.

5. Òscar Barbera Aresté, Àstrid Barrio López y Juan Rodríguez Teruel, op. cit., 2009, anexo 1.

6. Michel Martínez Pérez, Chunta Aragonesista (CHA): una historia aragonesa, Zaragoza, Cuadernos del Ebro, Fundación Gaspar Torrente, 2017, p. 99-115.

7. Carlos Serrano Lacarra, “Socialismo con denominación de origen: el PSA y sus 'secuelas”, in IV Congreso de Historia Local de Aragón (Barbastro, 3-5 de julio de 2003), Barbastro, Instituto de Estudios Altoaragoneses : Universidad Nacional de Educación a Distancia, UNED, 2005, p. 245-260.

8. Hipólito Gómez de las Roces fue presidente de la Diputación Provincial de Zaragoza entre 1974 y 1977.

9. El primer lema del PAR en 1978 fue explícito: "Las cosas claras: España es nuestra patria, Aragón nuestro partido".

10. Aragón es también una comunidad foral con derecho civil propio.

11. En las elecciones europeas de 1994, el PAR se presenta junto al PNV y Unió Mallorquina, entre otros, formando la lista "Coalición nacionalista". Aquel año, CiU se presentó en solitario.

12. El Partido Aragonés Regionalista (PAR) se convierte en Partido Aragonés (Par).

13. Chunta Aragonesista en aragonés, Unión Aragonesista (castellano) y Unió Aragonesista (catalán) entre 1986 y 1991, primeros tiempos de una denominación trilingüe.

14. Se da además la circunstancia que los electores aragoneses han votado siempre como el conjunto de los españoles. El País habló de Aragón como del Ohio español. https://elpais.com/ politica/2015/10/08/actualidad/1444335805_496770.html, consultada el 4 de octubre de 2018.

15. Sólo obtiene algunos concejales en el norte pirenaico gracias a su programa ecologista opuesto a la construcción de embalses que inundarían valles y pueblos pirenaicos. Los casos de Biscarrués o Jánovas ilustran ese "colonialismo interior" que denuncian los aragonesistas desde el tardofranquismo.

16. En 1999, CHA pasa de dos diputados en Cortes de Aragón a cinco mientras que, en 2003, obtiene hasta nueve. http://www.historiaelectoral.com/aaragon.html, página consultada el 4 de octubre de 2018.

17. José Antonio Labordeta es diputado de CHA en el Congreso de los Diputados entre 2000 y 2008 , es decir durante la última legislatura de José María Aznar (PP, con mayoría absoluta) y la primera de José Luis Rodríguez Zapatero (PSOE, con mayoría relativa).

18. En 2007, CHA pierde más de la mitad de sus votantes en las elecciones municipales de Zaragoza con respecto a 2003, pasando de 62211 votos (el 18,82 \%) en 2003 a 29230 votos (el 9,89 \%) en 2007. Cf. Michel Martinez Pérez, op. cit., 2017, p. 267.

19. Ibid., p. 291.

20. https://chesusyuste.wordpress.com/2015/07/01/el-acuerdo-de-investidura-psoe-cha-50medidas-para-un-gobierno-de-izquierdas-en-aragon/, consultada el 4 de octubre de 2018. Así lo contó en su blog, el exdiputado en Cortes de Aragón y Congreso de los Diputados, Chesús Yuste. 
De entre los 50 puntos, se pueden destacar el ingreso de inserción básica, la derogación de la LOMCE, la moratoria de los desahucios, la lucha contra la pobreza energética, la ley de lenguas y el blindaje del Estatuto de autonomía. Notamos, por tanto, un pacto de gobierno con marcado perfil social y aragonesista.

21. En octubre de 2017, el gobierno de Aragón aprobó la Directriz Especial de Política Demográfica y contra la Despoblación, para tratar de frenar este grave problema que comparte Aragón con otras CC.AA. del interior peninsular o regiones del extremo norte europeo (Laponia, High Lands de Escocia...). http://www.aragon.es/DepartamentosOrganismosPublicos/ Departamentos/VertebracionTerritorioMovilidadVivienda/AreasTematicas/

OrdenacionTerritorial/PoliticaDespoblacion/

ci.txt_despoblacion_documentos.detalleDepartamento?channelSelected $=0$, consultada el 4 de octubre de 2018. https://www.eldiario.es/aragon/sociedad/Aragon-cuenta-directriz-especificadespoblacion_0_703080537.html, consultada el 4 de octubre de 2018.

22. En el anterior gobierno PP-Par, hubo dos consejeros para gestionar estas carteras.

23. El trayecto Zaragoza-Teruel es de $2 \mathrm{H} 35$ minutos mientras que, en coche, por la autovía A23 puede realizarse el trayecto en $1 \mathrm{H} 45$ minutos. La misma diferencia se puede apreciar entre Teruel y Valencia. En octubre de 2018, también se hizo viral el vídeo de un tractor que adelantaba al tren en la provincia de Teruel.

http://cadenaser.com/ser/2018/09/13/sociedad/1536828335_490748.html, consultada el 4 de octubre de 2018.

24. https://www.elperiodicodearagon.com/noticias/economia/gm-deja-utilizar-linea-ferreateruel-transportar-coches_1033945.html, consultada el 4 de octubre de 2018.

25. https://www.fomento.gob.es/el-ministerio/sala-de-prensa/noticias/mie-06062018-1606, consultada el 4 de octubre de 2018.

26. http://www.aragonradio.es/noticias/hemeroteca/al-menos-16-autobuses-viajaran-de-teruela-la-movilizacion-por-el-tren-del-7-de-octubre-en-valencia, consultada el 4 de octubre de 2018.

27. https://www.eleconomista.es/economia/noticias/9416165/09/18/Abalos-promete-elCorredor-Mediterraneo-en-2021-ante-las-reservas-empresariales.html, consultada el 4 de octubre de 2018.

28. https://www.lacomarca.net/conoce-tu-pais-y-cuentalo/, consultada el 4 de octubre de 2018.

29.

https://www.heraldo.es/noticias/aragon/

2015/03/23/10_los_pueblos_mas_bonitos_espana_estan_aragon_346830_300.html, consultada el 4 de octubre de 2018.

30. LEY 8/2018, de 28 de junio, de actualización de los derechos históricos de Aragón.

31. Así lo defiende Miguel Martínez Tomey, responsable de los asuntos europeos de CHA, en un artículo en Heraldo de Aragón publicado el 23 de agosto de 2018 . http:// comunicacioninterna.chunta.org/wordpress/?p=4040 Página consultada el 4 de octubre de 2018. También la defendió el Consejero Soro en una entrevista al diario.es https://www.eldiario.es/ aragon/politica/Ciudadanos-PP-Ley-Derechos-Historicos_0_792321711.html, consultada el 4 de octubre de 2018.

32. De ahí surgieron los famosos acrónimos LAPAO y LAPAPYP que no aparecían en la ley.

33. https://www.elperiodicodearagon.com/noticias/aragon/ley-lenguas-llega-tribunalconstitucional_875781.html, consultada el 4 de octubre de 2018.

34. https://www.heraldo.es/noticias/aragon/2016/03/18/el-constitucional-valida-ley-lenguasaragon-pesar-nombrar-catalan-827053-300.html, consultada el 4 de octubre de 2018.

35. LEY 3/1999, de 10 de marzo, del Patrimonio Cultural Aragonés.

36. http://www.lenguasdearagon.org/direccion-general-de-politica-linguistica-2/, consultada el 3 octubre 2018.

37. Los motivos son obviamente políticos y están sujetos a los vaivenes de la política española, y también aragonesa, donde el anticatalanismo es utilizado por partidos políticos para captar 
votantes. La oficialización de la lengua catalana en una parte de Aragón ha sido una decisión política inasumible para muchos partidos.

\section{RESÚMENES}

En 2015, con la crisis del bipartidismo en España, los resultados de los comicios autonómicos dividieron aún más unas Cortes de Aragón tradicionalmente multipartidistas. A los partidos de ámbito estatal (PP, PSOE e IU) se sumaron las nuevas propuestas de Podemos y Ciudadanos mientras que los partidos de ámbito territorial, Partido Aragonés (Par) y Chunta Aragonesista (CHA), mantuvieron su presencia parlamentaria en las Cortes de Aragón. En el Palacio de la Aljafería, sede del parlamento aragonés, se alcanzó una mayoría progresista (35 diputados con la suma de PSOE, Podemos, CHA e IU) frente a los 32 diputados del bloque conservador (PP, Ciudadanos y Par). Este resultado permitió una coalición inédita en el poder ejecutivo (PSOECHA) con el apoyo parlamentario puntual de Podemos e Izquierda Unida. Veremos cómo los nacionalistas de CHA, aunque debilitados, han logrado imponer, desde una sola consejería del gobierno autonómico, temáticas cruciales de su agenda aragonesista: derechos históricos, vertebración del territorio, política lingüística...

En 2015, dans un contexte de crise pour le bipartisme PP-PSOE en Espagne, les résultats des élections « autonomiques » ont éclaté encore un peu plus les Cortès d'Aragon traditionnellement multipartites. Aux partis d'obédience nationale (PP, PSOE, et IU) se sont greffées les nouvelles propositions de Podemos et Ciudadanos tandis que les partis autonomistes, Partido Aragonés (PAR) et Chunta Aragonesista (CHA), ont maintenu leur présence parlementaire aux Cortès aragonaises. Au Palais de la Aljafería, siège du parlement aragonais, une majorité de gauche progressiste s'est dessinée (35 députés de PSOE, Podemos, CHA et IU) contre 32 pour le bloc conservateur (PP, PAR et Ciudadanos). Ce résultat a donné lieu à une coalition inédite pour le pouvoir exécutif aragonais (PSOE-CHA) ponctuellement soutenue au niveau législatif par Podemos et Izquierda Unida. Il sera question de voir comment les nationalistes de CHA, pourtant très affaiblis, ont réussi à imposer avec un seul ministère régional ("consejería") des thématiques cruciales de l'agenda politique nationaliste: droits historiques, articulation du territoire, politique linguistique...

\section{ÍNDICE}

Mots-clés: aragonesismo, politique linguistique d'Aragón, coalition PSOE-CHA, droits historiques, articulation

Palabras claves: aragonesismo, política lingüística de Aragón, coalición PSOE-CHA, derechos históricos, vertebración

\section{AUTOR}

\section{MICHEL MARTÍNEZ PÉREZ}

Université Toulouse Capitole, FRAMESPA UMR 5136/CRIMIC EA 2561 